\title{
UNIQUENESS FOR A FORWARD BACKWARD DIFFUSION EQUATION
}

BY

ALAN V. LAIR

\begin{abstract}
Let $\phi$ be continuous, have at most finitely many local extrema on any bounded interval, be twice continuously differentiable on any closed interval on which there is no local extremum and be strictly decreasing on any closed interval on which it is decreasing. We show that the initial-boundary value problem for $u_{t}=\phi\left(u_{x}\right)_{x}$ with Neumann boundary conditions has at most one smooth solution.
\end{abstract}

Introduction. Recently, in a series of papers [3-6], Höllig and Nohel considered the nonlinear diffusion equation

$$
u_{t}=\phi\left(u_{x}\right)_{x}
$$

in which $\phi$ is nonmonotonic. The motivation for studying (0.1) springs from the Clausius-Duhem inequality [1, p. 79] which, in one space dimension, implies that the flux, $\phi\left(u_{x}\right)$, need not be a monotonic function of the temperature gradient, $u_{x}$, although it does require that $u_{x}$ and $\phi\left(u_{x}\right)$ have the same sign. In view of this, the authors in $[3,4]$ require that the less restrictive coercivity condition

$$
s \phi(s) \geq c s^{2}, \quad c>0, s \in R=\text { the reals, }
$$

be satisfied.

In [5, 6] Höllig and Nohel consider the Cauchy problem for $(0.1)$ in which $\phi(s)=\max \{s, 0\}$, so that $(0.2)$ holds for $s \geq 0$, and derive results about the form of the free boundary $s(t)$ where $u_{x}\left(s(t)^{+}, t\right)=0$. In [4] the well-posedness of the initial-boundary value problem for $(0.1)$ on $D \equiv[0,1] \times[0, T]$ is discussed in the context of homogeneous Neumann boundary conditions. An especially interesting result in [4] is a maximum-minimum principle which, among other things, yields the result that $\phi^{\prime}\left(u_{x}\right)$ is positive on $D$ provided that $\phi^{\prime}\left(f^{\prime}\right)$ is positive on $[0,1]$ where $f$ is the initial temperature distribution, and $u$ is a smooth $\left(W^{2,2}\right)$ solution of $(0.1)$. Thus if $f$ is smooth and $\phi^{\prime}\left(f^{\prime}\right)$ is positive on $[0,1]$, then the initial-boundary value problem for $(0.1)$ can be treated as an ordinary (i.e. forward-in-time) diffusion process. If, however, $\phi^{\prime}\left(f^{\prime}\right)$ is negative for some $x$, then $\phi^{\prime}\left(u_{x}(x, t)\right)$ is negative for all $t$ near zero, and equation (0.1) corresponds to a backward diffusion equation near the point $(x, 0)$. In this case, Höllig [3] has shown that for continuous piecewise linear $\phi$ satisfying (0.2), $C^{2}$ initial temperature distribution $f$ and homogeneous Neumann boundary conditions, the initial-boundary value problem for $(0.1)$ has infinitely many (nonsmooth) solutions. These solutions have highly discontinuous derivatives

Received by the editors July 6, 1984 and, in revised form, November 20, 1984. 1980 Mathematics Subject Classification. Primary 35K55, 35K65.

(1985 American Mathematical Society $0002-9947 / 85 \$ 1.00+\$ .25$ per page 
and as pointed out in [3], if $f$ is not analytic, this is to be expected since no solution of the initial-boundary value problem studied in [3] can have $u_{x}$ piecewise continuous with respect to any finite partition of $D$.

The purpose of this paper is to prove that the intial-boundary value problem for (0.1) with Neumann boundary conditions (not necessarily homogeneous) and smooth initial condition has at most one smooth solution provided $\phi$ satisfies the following conditions:

(A) The function $\phi$ is continuous on $R$ and has at most finitely many local extrema on any bounded interval.

(B) If $r, s$ are adjacent local extrema of $\phi$ such that $r<s$, then $\phi \in C^{2}([r, s])$.

(C) If $\phi^{\prime}(s)<0$ for all $s \in(a, b)$, then $\phi^{\prime}\left(a^{+}\right) \cdot \phi^{\prime}\left(b^{-}\right) \neq 0$.

It is clear that assumption (C) implies that $\phi^{\prime}$ fails to exist at all local extreme points. The specific examples of $\phi$ analyzed earlier in $[3,5,6]$ have this property and indeed satisfy all of the above restrictions. Some of the analysis in [4] deals with specific examples that also satisfy them although the a priori estimates included in [4] would apply to more general forms of $\phi$.

Main result. We let $C_{*}(D)$ denote the set of functions for which $u, u_{x}, u_{t}$ and $u_{x x}$ are continuous on $D, u_{x t} \in L_{\infty}(D)$ and $u_{x t}$ is continuous a.e. on $D$. Although, in general, the initial temperature distribution $f$ may need to be quite smooth to ensure the possibility of the existence of a solution to our problem (e.g. $f$ must be analytic if $\phi^{\prime}$ is smooth and negative on $R$ ), we shall state our results using only the restrictions on $f$ which are necessary to ensure the correctness of our proof. The main result of this paper is the proof of the following theorem.

TheOrem. Suppose $u, v \in C_{*}(D)$ and satisfy $(0.1)$ on $D$ as well as

$$
\begin{aligned}
u(x, 0) & =f(x), \quad x \in[0,1], \\
u_{x}(0, t) & =h_{0}(t), \quad u_{x}(1, t)=h_{1}(t), \quad t \in[0, T],
\end{aligned}
$$

where $f \in C^{1}([0,1])$ and $h_{0}, h_{1} \in C([0, T])$ are known functions. Then $u=v$ on $D$.

Before proving this assertion, we need some preliminary results. Also, for the remainder of the paper, we assume that $u, v, f, h_{0}, h_{1}$ satisfy the hypothesis of the theorem.

Let $A=\min \left\{\min _{D} u_{x}, \min _{D} v_{x}\right\}$ and $B=\max \left\{\max _{D} u_{x}, \max _{D} v_{x}\right\}$. Clearly the ranges of the functions $u_{x}, v_{x}, h_{0}, h_{1}, f^{\prime}$ are contained in the closed interval $[A, B]$. We let $m_{1}, m_{2}, \ldots, m_{n-1}$ be the points in the open interval $(A, B)$ where $\phi$ has local extrema. (If $\phi$ has no local extremum, then there is nothing to prove.) Let $m_{0}=A$, $m_{n}=B$ and assume that $m_{0}<m_{1}<\cdots<m_{n}$. Let $I_{j}$ be the open interval $\left(m_{j-1}, m_{j}\right)$ and $\bar{I}_{j}$ the closed interval $\left[m_{j-1}, m_{j}\right], j \in\{1,2, \ldots, n\}$. We now define functions $p^{j}, q^{j}, r^{j}, s^{j}$ on $D ; h_{0}^{j}, h_{1}^{j}$ on $[0, T]$ and $f^{j}, g^{j}$ on $[0,1], j \in\{1, \ldots, n\}$, as follows:

$$
p^{j}(x, t)= \begin{cases}u_{x}(x, t) & \text { if } u_{x}(x, t) \in I_{j}, \\ m_{j-1} & \text { if } u_{x}(x, t) \leq m_{j-1}, \\ m_{j} & \text { if } u_{x}(x, t) \geq m_{j}\end{cases}
$$




$$
q^{j}(x, t)= \begin{cases}u_{t}(x, t) & \text { if } u_{x}(x, t) \in I_{j} \\ 0 & \text { if } u_{x}(x, t) \notin I_{j}\end{cases}
$$

By replacing $u$ in the definitions of $p^{j}$ and $q^{j}$ with $v$, we obtain the definitions of $r^{j}$ and $s^{j}$, respectively. Also, we let

$$
h_{0}^{j}(t)= \begin{cases}h_{0}(t) & \text { if } h_{0}(t) \in I_{j}, \\ m_{j-1} & \text { if } h_{0}(t) \leq m_{j-1}, \\ m_{j} & \text { if } h_{0}(t) \geq m_{j} .\end{cases}
$$

By replacing $h_{0}$ in the definition of $h_{0}^{j}$ with $h_{1}$ and then with $f^{\prime}$, we obtain the definitions of $h_{1}^{j}$ and then $g^{j}$, respectively. Finally, let $f^{j}(x)=\int_{0}^{x} g^{j}(\alpha) d \alpha$.

Lemma 1. Assume the same hypothesis as the theorem. If $u_{x}\left(x_{0}, t_{0}\right) \in$ $\left\{m_{0}, m_{1}, \ldots, m_{n}\right\}$ and $0<x_{0}<1$, then $u_{x x}\left(x_{0}, t_{0}\right)=u_{t}\left(x_{0}, t_{0}\right)=0$. The same result holds for $v$.

Proof. Suppose $u_{x}\left(x_{0}, t_{0}\right)=m_{j}$ for some $j \in\{0,1, \ldots, n\}$. If $u_{x}\left(x, t_{0}\right) \leq m_{j}$ ( $\geq m_{j}$ ) for all $x$ near $x_{0}$, then $u_{x}\left(\cdot, t_{0}\right.$ ) has a local maximum (minimum) at $x_{0}$ and thus $u_{x x}\left(x_{0}, t_{0}\right)=0$. Then since $u$ satisfies $(0.1)$, we immediately obtain $u_{t}\left(x_{0}, t_{0}\right)=$ 0 . If $u_{x}\left(\cdot, t_{0}\right)-m_{j}$ takes on both positive and negative values in every neighborhood of $x_{0}$, then we obtain

$$
u_{t}\left(x_{0}, t_{0}\right)=\phi^{\prime}\left(m_{j}^{+}\right) u_{x x}\left(x_{0}, t_{0}\right)=\phi^{\prime}\left(m_{j}^{-}\right) u_{x x}\left(x_{0}, t_{0}\right)
$$

from (0.1). Thus, since condition (C) implies that $\phi^{\prime}\left(m_{j}^{+}\right) \neq \phi^{\prime}\left(m_{j}^{-}\right)$, the desired result follows.

Lemma 2. Assume the same hypothesis as the theorem. For each $j \in\{1,2, \ldots, n\}$, we have

$$
\left|p^{j}(x, t)-p^{j}\left(x_{0}, t_{0}\right)\right| \leq\left|u_{x}(x, t)-u_{x}\left(x_{0}, t_{0}\right)\right| \text { on } D \text {. }
$$

Also, for every $\left(x_{0}, t_{0}\right) \in(0,1) \times[0, T]$, there exists a $\delta\left(x_{0}, t_{0}\right)>0$ such that

$$
\left|q^{j}(x, t)-q^{j}\left(x_{0}, t_{0}\right)\right| \leq\left|u_{t}(x, t)-u_{t}\left(x_{0}, t_{0}\right)\right|
$$

whenever $(x, t) \in D$ and $\left(x-x_{0}\right)^{2}+\left(t-t_{0}\right)^{2}<\delta^{2}\left(x_{0}, t_{0}\right)$. Similar results hold for the functions $r^{j}$ and $s^{j}$.

Proof. The proof of inequality (1.2) is easy and therefore omitted. Thus we prove (1.3) only. It is clear that if both $u_{x}(x, t)$ and $u_{x}\left(x_{0}, t_{0}\right)$ are in $I_{j}$ or if neither is in $I_{j}$, then (1.3) holds trivially. Also, if $u_{x}\left(x_{0}, t_{0}\right) \in I_{j}$ or $u_{x}\left(x_{0}, t_{0}\right) \notin \bar{I}_{j}$, then $\delta\left(x_{0}, t_{0}\right)$ may be chosen sufficiently small that $u_{x}(x, t) \in I_{j}$ or $u_{x}(x, t) \notin \bar{I}_{j}$ whenever $\left(x-x_{0}\right)^{2}+\left(t-t_{0}\right)^{2}<\delta^{2}\left(x_{0}, t_{0}\right)$. Thus, inequality (1.3) holds trivially for $\left(x-x_{0}\right)^{2}+\left(t-t_{0}\right)^{2}<\delta^{2}\left(x_{0}, t_{0}\right)$. Therefore the only significant difficulty in proving (1.3) seems to occur when $u_{x}\left(x_{0}, t_{0}\right) \in\left\{m_{j-1}, m_{j}\right\}$. However, in this case we apply Lemma 1 to obtain $u_{t}\left(x_{0}, t_{0}\right)=0$ so that (1.3) is equivalent to proving $\left|q^{j}(x, t)\right| \leq\left|u_{t}(x, t)\right|$ which is trivially true. 
Lemma 3. Assume the same hypothesis as the theorem. For each $j \in\{1,2, \ldots, n\}$, the derivatives $p_{t}^{j}$ and $q_{x}^{j}$ exist a.e., are essentially bounded and equal a.e. on $D$. The same result holds for $r_{t}^{j}$ and $s_{x}^{j}$.

Proof. Fix $j \in\{1,2, \ldots, n\}$ and let $G=\left\{(x, t) \in D \mid u_{x}(x, t) \notin I_{j}\right\}$. It suffices to prove the result a.e. on $G$ since $u_{x t}=p_{t}^{j}=q_{x}^{j}$ a.e. on the complement of $G$. Also, it is clear that in the interior of $G$, we have trivially that $p_{t}^{j}=q_{x}^{j}=0$. Thus we need only prove the result on the boundary of $G, \partial G$. Note that $u_{x}(x, t) \in\left\{m_{j-1}, m_{j}\right\}$ for all $(x, t) \in \partial G$. In addition, since $u_{x t}$ exists and is continuous a.e. on $D$, we need only prove the result on the set $\partial G^{\prime} \equiv\left\{(x, t) \in \partial G \mid u_{x t}\right.$ is continuous at $\left.(x, t)\right\}$. We let $\partial G^{\prime}=G_{1} \cup G_{2}$, where $G_{1} \equiv\left\{(x, t) \in \partial G^{\prime} \mid u_{x t}(x, t) \neq 0\right\}$ and $G_{2}=\{(x, t) \in$ $\left.\partial G^{\prime} \mid u_{x t}(x, t)=0\right\}$. To prove our result, we shall show that $G_{1}$ has measure zero and that the desired equality holds at every point in $G_{2}$.

To show that $G_{1}$ has measure zero, it suffices to show that every closed subset of $G_{1}$ has measure zero. Thus let $F$ be any closed and therefore compact subset of $G_{1}$. Using analysis very similar to that in the proof of the Implicit Function Theorem [9, p. 236], it is easy to show that for each $\left(x_{0}, t_{0}\right) \in F$, there is an open rectangle $R_{0} \equiv\left(a_{0}, b_{0}\right) \times\left(c_{0}, d_{0}\right)$ containing $\left(x_{0}, t_{0}\right)$ such that $V_{0} \equiv\left\{(x, t) \in R_{0} \mid u_{x}(x, t) \in\right.$ $\left.\left\{m_{j-1}, m_{j}\right\}\right\}$ is a function ( $t$ is a function of $x$ ) and, in fact, continuous. Since this can be done at each point in $F$, the set of all such open rectangles forms an open covering of $F$. Let $\left\{R_{0}^{1}, \ldots, R_{0}^{\alpha}\right\}$ be a finite subcover and let $V_{0}^{i}=\{(x, t) \in$ $\left.R_{0}^{i} \mid u_{x}(x, t) \in\left\{m_{j-1}, m_{j}\right\}\right\}$. Then $F=\bigcup_{i=1}^{\alpha} V_{0}^{i}$. However, each set $V_{0}^{i}$ has measure zero (see [9, p. 273]) and hence $F$ has measure zero.

Now suppose $\left(x_{0}, t_{0}\right) \in G_{2}$. We wish to show that $p_{t}^{j}\left(x_{0}, t_{0}\right)=q_{x}^{j}\left(x_{0}, t_{0}\right)=0$. Lemma 2 implies that $\left|p^{j}\left(x_{0}, t\right)-p^{j}\left(x_{0}, t_{0}\right)\right| \leq\left|u_{x}\left(x_{0}, t\right)-u_{x}\left(x_{0}, t_{0}\right)\right|$ on $D$ so that dividing each side of this inequality by $\left|t-t_{0}\right|$ and letting $t \rightarrow t_{0}$, we observe that the right side, and hence the left side, approaches zero since $\left(x_{0}, t_{0}\right) \in G_{2}$. Thus $p_{t}^{j}\left(x_{0}, t_{0}\right)=0$. Similarly $q_{x}^{j}\left(x_{0}, t_{0}\right)=0$ for $0<x_{0}<1$. Thus $p_{t}^{j}=q_{x}^{j}$ a.e. on $D$. Note also that we have shown that $\left|u_{x t}\right|$ is an upper bound for both $\left|p_{t}^{j}\right|$ and $\left|q_{x}^{j}\right|$ a.e. on $D$. Thus $p_{t}^{j}, q_{x}^{j} \in L_{\infty}(D)$.

Lemma 4. Assume the same hypothesis as the theorem. For each $j \in\{1,2, \ldots, n\}$, there exist functions $u^{j}, v^{j} \in C^{\prime}(D)$ such that $u_{x}^{j}=p^{j}, u_{t}^{j}=q^{j}, v_{x}^{j}=r^{j}, v_{t}^{j}=s^{j}$ on $D$.

Proof. (Although Green's Theorem in its usual form (see e.g. [9, p. 482]) cannot be directly applied to obtain this result because of the lack of smoothness of $p^{j}$ and $q^{j}$, the proof here is very similar and therefore omitted.)

Lemma 5. Assume the same hypothesis as the theorem. For each $j \in\{1,2, \ldots, n\}$ the functions $u^{j}, v^{j}$ defined in Lemma 4 satisfy

$$
\begin{aligned}
u_{t}^{j} & =\phi\left(u_{x}^{j}\right)_{x}, \quad 0<x<1,0 \leq t \leq T, \\
u_{x}^{j}(0, t) & =h_{0}^{j}(t), \quad u_{x}^{j}(1, t)=h_{1}^{j}(t), \quad 0 \leq t \leq T, \\
u^{j}(x, 0) & =f^{j}(x), \quad 0 \leq x \leq 1 .
\end{aligned}
$$


Proof. Fix $j \in\{1,2, \ldots, n\}$. Equations (1.5) are easily verified from the definitions of $u^{j}, v^{j}$ and the fact that $u, v$ satisfy equations (1.1). It remains only to prove (1.4). Again, since the proofs are quite similar, we prove (1.4) holds for $u^{j}$ and omit the proof for $v^{j}$. Since $u_{x}^{j}=p^{j}=u_{x}$ and $u_{t}^{j}=q^{j}=u_{t}$ for $u_{x}(x, t) \in I_{j}$, it is clear that (1.4) holds for $u_{x}(x, t) \in I_{j}$. Also, $u_{t}^{j}=\phi\left(u_{x}^{j}\right)_{x}=0$ if $u_{x}(x, t) \notin I_{j}$ since, in this case, $u_{x}^{j}$ is constant in a neighborhood of such a point $(x, t)$. This leaves only the case $u_{x}\left(x_{0}, t_{0}\right) \in\left\{m_{j-1}, m_{j}\right\}$. For $0<x_{0}<1$, Lemma 2 implies that

$$
\left|\frac{u_{x}^{j}\left(x, t_{0}\right)-u_{x}^{j}\left(x_{0}, t_{0}\right)}{x-x_{0}}\right|=\left|\frac{p^{j}\left(x, t_{0}\right)-p^{j}\left(x_{0}, t_{0}\right)}{x-x_{0}}\right| \leq\left|\frac{u_{x}\left(x, t_{0}\right)-u_{x}\left(x_{0}, t_{0}\right)}{x-x_{0}}\right| .
$$

Since Lemma 1 implies $u_{x x}\left(x_{0}, t_{0}\right)=0$, the right side of (1.6) approaches zero as $x$ approaches $x_{0}$, and thus we get $u_{x x}^{j}\left(x_{0}, t_{0}\right)=0$. Thus, $\phi\left(u_{x}^{j}\right)_{x}=0$ at $\left(x_{0}, t_{0}\right)$. Since $u_{t}^{j}=q^{j}$ on $D$, it is obvious that $u_{t}^{j}\left(x_{0}, t_{0}\right)=0$ by the definition of $q^{j}$.

Lemma 6. Assume the same hypothesis as the theorem. The functions $u^{j}, v^{j}$ defined in Lemma 4 are equal for all $j \in\{1,2, \ldots, n\}$.

Proof. Fix $j \in\{1,2, \ldots, n\}$ and note that $u_{x}^{j}, v_{x}^{j} \in \bar{I}_{j}$ on $D$. Thus $\phi^{\prime}\left(u_{x}^{j}\right)$ and $\phi^{\prime}\left(v_{x}^{j}\right)$ do not switch signs on $D$. Therefore we need to consider two cases: Case 1 is $\phi^{\prime}\left(\bar{I}_{j}\right) \geq 0$ and Case 2 is $\phi^{\prime}\left(\bar{I}_{j}\right) \leq 0$.

Case $1\left(\phi^{\prime}\left(\bar{I}_{j}\right) \geq 0\right)$. Since $u^{j}, v^{j}$ satisfy (1.4) and (1.5), we have $\left(u^{j}-v^{j}\right)_{t}=$ $\left(\phi\left(u_{x}^{j}\right)-\phi\left(v_{x}^{j}\right)\right)_{x}$. Multiply this expression by $u^{j}-v^{j}$ and integrate with respect to $x$ over the closed interval $[0,1]$. After integrating by parts on the right side and using the fact that $u_{x}^{j}=v_{x}^{j}$ for $x \in\{0,1\}$, we get

$$
\int_{0}^{1}\left(u^{j}-v^{j}\right)\left(u^{j}-v^{j}\right)_{t}=-\int_{0}^{1}\left(u_{x}^{j}-v_{x}^{j}\right)\left(\phi\left(u_{x}^{j}\right)-\phi\left(v_{x}^{j}\right)\right) d x .
$$

However, since $u_{x}^{j}, v_{x}^{j} \in \bar{I}_{j}$ and $\phi^{\prime}\left(\bar{I}_{j}\right) \geq 0$, the right side of (1.7) is nonpositive. Thus (1.7) yields

$$
\frac{d}{d t} \int_{0}^{1}\left(u^{j}-v^{j}\right)^{2} d x \leq 0 .
$$

Now since $u^{j}=v^{j}$ at $t=0$, we may integrate (1.8) from 0 to $t$ to obtain

$$
\int_{0}^{1}\left(u^{j}(x, t)-v^{j}(x, t)\right)^{2} d x \leq 0
$$

which yields $u^{j}=v^{j}$ on $D$.

Case $2\left(\phi^{\prime}\left(I_{j}\right) \leq 0\right)$. By condition $(\mathrm{C})$, we observe that $\phi^{\prime}\left(\bar{I}_{j}\right) \leq 0$ implies that $\phi^{\prime}\left(\bar{I}_{j}\right)<0$. Thus, there exists a positive constant $\lambda$ such that $\phi^{\prime}(s) \leq-\lambda$ for all $s \in \bar{I}_{j}$. Define the function $h: D \rightarrow R$ as follows:

$$
h(x, t)= \begin{cases}\frac{\phi\left(u_{x}^{j}(x, t)\right)-\phi\left(v_{x}^{j}(x, t)\right)}{u_{x}^{j}(x, t)-v_{x}^{j}(x, t)} & \text { if } u_{x}^{j}(x, t) \neq v_{x}^{j}(x, t), \\ \phi^{\prime}\left(u_{x}^{j}(x, t)\right) \quad \text { if } u_{x}^{j}(x, t)=v_{x}^{j}(x, t) .\end{cases}
$$


It is clear that $h$ is continuous on $D$ since $u^{j}, v^{j} \in \bar{I}_{j}$. However, we also have that $h_{t}$ exists a.e. on $D$ and is essentially bounded. To see this, observe that the function $\beta$ : $R^{2} \rightarrow R$ defined by

$$
\beta(u, v)=\left\{\begin{array}{l}
\frac{\phi(u)-\phi(v)}{u-v} \quad \text { if } u \neq v \\
\phi^{\prime}(u) \text { if } u=v
\end{array}\right.
$$

has continuous (and thus bounded) first partials on $\bar{I}_{j}^{2}$ since $\phi^{\prime \prime}$ is bounded on $\bar{I}_{j}$ (see condition (B)) and Lemmas 3 and 4 imply that $u_{x t}^{j}, v_{x t}^{j} \in L_{\infty}(D)$. Now, letting $z=u^{j}-v^{j}$, we get that $z$ satisfies

$$
\begin{aligned}
& z_{t}=\left(h(x, t) z_{x}\right)_{x}, \quad 0<x<1,0 \leq t \leq T, \\
& z(x, 0)=0, \quad 0 \leq x \leq 1, \\
& z_{x}(0, t)=z_{x}(1, t)=0, \quad 0 \leq t \leq T,
\end{aligned}
$$

where $h<-\lambda<0, h$ is continuous on $D$ and $h_{t}$ is essentially bounded on $D$. Thus, using [7] or a modified form of [2, Theorem 6, p. 173], we get $z=0$ on $D$. (Both of the references $[2,7]$ assume homogeneous Dirichlet boundary conditions, but proofs work equally well for homogeneous Neumann boundary conditions.)

Proof of THE THEOREM. Let $W=\left\{(x, t) \in D \mid u_{x}(x, t)-v_{x}(x, t) \neq 0\right\}$. If $W=$ $\varnothing$, we have $u_{x}=v_{x}$ on $D$ and thus $u_{x x}=v_{x x}$ on $D$ which in turn implies that $u_{t}=v_{t}$. Thus, $u-v$ may be constant on $D$ which implies $u=v$ on $D$ since $u=v$ at $t=0$. Thus we need only prove $W=\varnothing$.

Therefore, suppose $W \neq \varnothing$. Let $(\bar{x}, \bar{y}) \in W$ and without loss of generality, we assume $u_{x}(\bar{x}, \bar{t})-v_{x}(\bar{x}, \bar{t})>0$. Let $\Omega$ be the largest open (in $D$ ) connected subset of $W$ containing $(\bar{x}, \bar{t})$. Thus $\Omega$ is the union of all open connected subsets of $W$ containing $(\bar{x}, \bar{t})$. Note that $u_{x}-v_{x}>0$ on $\Omega$. Since $\left\{\bar{I}_{j} \mid j=1, \ldots, n\right\}$ covers $[A, B]$, we have $u_{x}(\Omega) \cap \bar{I}_{k} \neq \varnothing$ for some $k$. Suppose $\left(x_{0}, t_{0}\right) \in \Omega$ such that $u_{x}\left(x_{0}, t_{0}\right) \in \bar{I}_{k}$. This implies that $u_{x}\left(x_{0}, t_{0}\right)=u_{x}^{k}\left(x_{0}, t_{0}\right)=v_{x}^{k}\left(x_{0}, t_{0}\right)$, the last equality being a consequence of Lemma 6. Now note that we must have $u_{x}\left(x_{0}, t_{0}\right)$ $=v_{x}^{k}\left(x_{0}, t_{0}\right) \in\left\{m_{k-1}, m_{k}\right\}$ for if $u_{x}\left(x_{0}, t_{0}\right) \in I_{k}$, then $v_{x}^{k}\left(x_{0}, t_{0}\right) \in I_{k}$ so that $v_{x}^{k}\left(x_{0}, t_{0}\right)=v_{x}\left(x_{0}, t_{0}\right)$. Therefore $u_{x}\left(x_{0}, t_{0}\right)=v_{x}\left(x_{0}, t_{0}\right)$ contradicting that $\left(x_{0}, t_{0}\right)$ $\in W$. Thus, given any $(x, t) \in \Omega$, we get $u_{x}(x, t) \in\left\{m_{0}, m_{1}, \ldots, m_{n}\right\}$. Since $\Omega$ is connected and $u_{x}$ is continuous, we get that $u_{x}$ is constant on $\Omega$. A similar argument will yield that $v_{x}$ is constant on $\Omega$. Therefore, we obtain $u_{x}-v_{x}=c$ (a positive constant) on $\Omega$. We must now show that $c$ is zero. However, this follows easily from the definition of $\Omega$ and the fact that $u_{x}-v_{x}$ vanishes on the lateral boundary of $D$. Thus we obtain $W=\varnothing$. This completes the proof.

REMARK. It should be noted that assumption (C), the most restrictive of the three conditions on $\phi$, could be omitted if the equation $u_{t}=\left(a(x, t) u_{x}\right)_{x}$ with homogeneous Neumann boundary conditions and homogeneous initial condition could be shown to have only the trivial solution for the backward, degenerate case $a \leq 0$ on $D$. 


\section{REFERENCES}

1. W. Day, The thermodynamics of simple materials with fading memory, Tracts in National Philosophy, vol. 22, Springer-Verlag, New York, Heidelberg and Berlin, 1970.

2. A. Friedman, Partial differential equations of parabolic type, Prentice-Hall, Englewood Cliffs, N. J., 1964. MR 31 \#6062.

3. K. Höllig, Existence of infinitely many solutions for a forward backward heat equation, Trans. Amer. Math. Soc. 278 (1983), 299-316.

4. K. Höllig and J. A. Nohel, $A$ diffusion equation with a nonmonotone constitutive function, Systems of Partial Differential Equations (J. M. Ball, ed.), Reidel, Dordrecht, 1983, pp. 409-422. MR 84k: 35082.

5. _ A singular free boundary problem, Texas A \& M Univ. Center for Approx. Theory Tech. Report \#41, 1983.

6. A A nonlinear integral equation occurring in a singular free boundary problem, Trans. Amer. Math. Soc. 283 (1984), 145-155.

7. A. V. Lair, Uniqueness of solutions of singular, backward-in-time parabolic equations and inequalities (to appear).

8. H. L. Royden, Real analysis, 2nd ed., Macmillan, New York, 1968.

9. A. E. Taylor and W. R. Mann, Advanced calculus, 2nd ed., xerox, Lexington, Mass., 1972.

Department of Mathematics, U. S. Air Force Institute of Technology, Wright - Patterson AFB, OHIO 45433 